\title{
Sudden falls as a persistent complication of selective dorsal rhizotomy surgery in children with bilateral spasticity: report of 3 cases
}

\author{
Laura R. Grootveld, MSc, ${ }^{1}$ Petra E. M. van Schie, PhD,, ${ }^{1,2}$ Annemieke I. Buizer, MD, PhD, ${ }^{1}$ \\ R. Jeroen Vermeulen, MD, PhD, ${ }^{3}$ Willem J. R. van Ouwerkerk, MD, PhD, ${ }^{4}$ \\ Rob L. M. Strijers, MD, PhD, ${ }^{5}$ and Jules J. G. Becher, MD, PhD ${ }^{1}$
}

Departments of ${ }^{1}$ Rehabilitation Medicine, ${ }^{2}$ Physical Therapy, ${ }^{4}$ Neurosurgery, and ${ }^{5}$ Clinical Neurophysiology, VU University Medical Center, Amsterdam; and ${ }^{3}$ Department of Child Neurology, Maastricht University Medical Centre, Maastricht, The Netherlands

\begin{abstract}
Selective dorsal rhizotomy (SDR) surgery is a well-established treatment for ambulatory children with bilateral spastic paresis and is performed to eliminate spasticity and improve walking. The objective of this case report is to describe sudden falls as a persistent complication of SDR. The authors report on 3 patients with bilateral spastic paresis, aged 12,6 , and 7 years at the time of surgery. The percentage of transected dorsal rootlets was around $40 \%$ at the L2-S1 levels. Sudden falls were reported with a frequency of several a day, continuing for years after SDR. The falls were often triggered by performing dual tasks as well as occurring in the transition from sitting to standing, during running, after strenuous exercise, or following a fright. Patients also had residual hyperesthesia and dysesthesia of the foot sole. The authors hypothesize that the sudden falls are caused by a muscle inhibition reflex of the muscles in the legs, as an abnormal reaction to a sensory stimulus that is perceived with increased intensity by a patient with hyperesthesia. A favorable effect of gabapentin medication supports this hypothesis.
\end{abstract}

http://thejns.org/doi/abs/10.3171/2016.2.PEDS15527

KEY WORDS selective dorsal rhizotomy; sudden falls; complication; bilateral spastic cerebral palsy; functional neurosurgery

$\mathrm{S}$ PASTICITY, one of the symptoms of spastic paresis, is a result of a neurological condition affecting the spinal cord or brain. Spasticity can be defined as hypertonia with one or both of the following signs: 1) resistance to externally imposed movement increases upon increasing speed of stretch and varies with the direction of joint movement; and 2) resistance to externally imposed movement rises rapidly above a threshold speed or joint angle. ${ }^{9}$ In childhood, about $80 \%$ of patients with cerebral palsy (CP) have a spastic motor disorder. CP is the most common motor disorder during childhood, with a prevalence of about 2 per 1000 live births. ${ }^{4,8}$ In patients with bilateral spastic CP, the most common MRI abnormality is periventricular leucomalacia. ${ }^{3}$ Another group of patients who often suffer from a high degree of spasticity is children with hereditary spastic paraplegia (HSP). HSP is a syndrome designation describing inherited disorders in which lower-extremity weakness and spasticity are the predominant symptoms, with no general abnormalities on MRI. Its prevalence has been estimated to range from 1.2 to 9.6 per $100,000 .^{2}$ More than 50 types of genetic deficits have been described as causing HSP. ${ }^{2}$

A specific group of ambulatory children with scores of I to III on the Gross Motor Function Classification System (GMFCS) and spastic paresis is eligible for selective dorsal rhizotomy (SDR) as a an effective method of reducing spasticity. ${ }^{11}$ Several techniques of SDR have been described. ${ }^{6}$ In our clinic, we perform SDR according to Peacock's method, ${ }^{7}$ in which a midline laminotomy is performed from L-2 to L-5, followed by selectively cutting some of the lumbosacral sensory spinal nerve rootlets. Before root testing and transection, sensory root S-2 is tested for bladder function by stimulating the penis or clitoris and recording a sensory evoked response in the root.

ABBREVIATIONS BMI = body mass index; $\mathrm{CP}=$ cerebral palsy; GABA = gamma-aminobutyric acid; GMFCS = Gross Motor Function Classification System; GMFM-66 = Gross Motor Function Measure-66; HSP = hereditary spastic paraplegia; SDR = selective dorsal rhizotomy.

SUBMITTED September 3, 2015. ACCEPTED February 8, 2016.

INCLUDE WHEN CITING Published online April 22, 2016; DOI: 10.3171/2016.2.PEDS15527. 
If a rootlet of S-1 shows an evoked response, this rootlet is preserved to prevent bladder impairment. Muscular responses are measured both with electromyography and manual palpation after electrical stimulation of a rootlet. The selection for transection is made based on the electrical threshold for muscular responses and the eradication of muscular responses in relation to the spinal level of the rootlet. Averaged over the treated levels, a maximum of $50 \%$ of the rootlets are cut. SDR reduces the sensory input from the lower limbs entering the spinal cord via the lumbosacral posterior nerve rootlets.

Long-term results of SDR have shown improvements in gross motor function and long-term functional benefits, without relapse of spasticity. ${ }^{1,5,10}$ At VU University Medical Center Amsterdam, the Netherlands, almost all 103 patients who have undergone SDR since 1998 have had an annual follow-up examination. All of these annual follow-up examinations were performed by one physician, who noticed that several patients experienced sudden falls shortly after SDR, although in most of them the problem fully resolved within months. Some of the patients, however, continued to experience these sudden falls after SDR or developed them later on. In this report, we describe 3 of these patients with persistent sudden falls. Their patient files were retrospectively analyzed. There were no significant differences between these 3 patients and the other 100 patients with respect to baseline demographic characteristics. Sudden falls were not present during the interval between initial presentation and surgery. To the best of our knowledge, sudden falls as a persistent complication of SDR have not previously been reported. The aim of this report is to record sudden falls as a persistent complication of SDR and draw attention to this complication.

\section{Case Reports}

Case 1

This male patient, who was 23 years old at the time of his most recent follow-up evaluation, was born prematurely and had bilateral spastic CP (baseline GMFCS Level II). He had MRI evidence of periventricular leukomalacia and a ventriculoperitoneal shunt for the treatment of hydrocephalus. At the age of 12 years, he underwent SDR at the L2-S1 levels, with an average of $44 \%$ of the dorsal rootlets transected on the left and $43 \%$ on the right. His body mass index (BMI) was within the normal range at the time of surgery as well as at the most recent follow-up (for patient characteristics, see Table 1). Just before surgery, his Gross Motor Function Measure-66 (GMFM-66) score was 70.4. Six months after SDR, he had recovered to this same level of functioning. The most recent GMFM-66 score was 64.3; this score was obtained when the patient was 21 years old, i.e., 9 years after SDR, thus showing a decrease in gross motor function compared with the situation before SDR. At physical examination, he reported hyperesthesia of the sole of his foot and his balance was normal. As of the most recent follow-up visit, in 2014, he had had no relapse of spasticity.

From 18 months after SDR until the latest follow-up 11 years after the operation, at the age of 23 , the patient reported sudden falls 10 to 15 times a day during the per- formance of dual tasks or the transition from sitting to standing. The sudden falls could also happen after a fright. The patient was not injured by the sudden falls. History and physical examination showed no indication for further investigations.

\section{Case 2}

This boy, who was 14 years old at his most recent follow-up examination, had bilateral spastic paresis that was probably due to HSP (baseline GMFCS Level II). MRI of the brain showed no abnormalities. DNA testing for $S P G 2$, $S P G 3 a, S P G 4, S P G 7$, and SPG31 genes showed no mutation. At the age of 6 years, he underwent SDR at L2-S1, with an average of $50 \%$ of the dorsal rootlets transected on the left and $47 \%$ on the right, because of a decrease in the range of motion of the ankle, knee, and hip. His BMI was within the normal range (see Table 1). Just before surgery, his GMFM-66 score was 81.93, and 3 years after SDR it was 85.23. His most recent GMFM-66 score was 89.70; this score was obtained when the patient was 13 years old, i.e., 7 years after SDR, showing an improvement in gross motor function compared with the situation before SDR. At physical examination he reported hyperesthesia of the soles of his feet; his balance was normal. As of the most recent follow-up visit, in 2014, he had had no relapse of spasticity.

One year after SDR, the patient reported sudden falls every couple of minutes during running or walking. Seven years after SDR, at the age of 14, the sudden falls were still occurring several times a day. He occasionally was injured by such a sudden fall. History and physical examination showed no indication for further investigations. To treat the hyperesthesia, he was prescribed $100 \mathrm{mg}$ gabapentin twice daily. This treatment effectively reduced the frequency of his sudden falls. However, it was discontinued due to side effects (emotional instability, anxiety, and obsessive behavior), after which the sudden falls became more frequent again.

\section{Case 3}

This young girl, who was 11 years old at her most recent follow-up examination, had bilateral spastic paresis that was probably due to HSP and was more severe on the right than the left (baseline GMFCS Level I). MRI of the brain showed no abnormalities. No DNA testing was performed. At the age of 7 years, the patient underwent SDR at the L2-S1 levels, with an average of 38\% dorsal rootlets transected on the left and $42 \%$ on the right. Her BMI was within the normal range (see Table 1). Her GMFM-66 score was 88.0 just before surgery and was unchanged 3 years after SDR. The most recent GMFM-66 score was 86.5 at the age of 11, i.e., 4 years after SDR, showing a decrease in gross motor function compared with her function before SDR. At physical examination she reported hyperesthesia of the L-3, L-4, L-5, and S-1 dermatomes; the hyperesthesia was more severe on the left side than on the right. Her balance was normal. As of the most recent follow-up visit, in 2015, she had had no relapse of spasticity.

Three months after SDR, the patient reported that she occasionally felt a vibration around her knees and would fall shortly thereafter. After such a sudden fall, she would 
TABLE 1. Characteristics of the 3 patients at the time of selective dorsal rhizotomy surgery

\begin{tabular}{|c|c|c|c|}
\hline Characteristic & Case 1 & Case 2 & Case 3 \\
\hline Sex & Male & Male & Female \\
\hline Age in yrs at SDR & 12 & 6 & 7 \\
\hline $\mathrm{BMI}\left(\mathrm{kg} / \mathrm{m}^{2}\right)$ at SDR & 19.6 & 15.0 & 17.0 \\
\hline GMFCS level at baseline & II & II & I \\
\hline GMFM-66 score at baseline & 70.4 & 81.9 & 88.0 \\
\hline Brain MRI findings & PVL, hydrocephalus, VPS & No abnormalities & No abnormalities \\
\hline Level of SDR & L2-S1 & L2-S1 & L2-S1 \\
\hline \multicolumn{4}{|c|}{ Percentage of dorsal rootlets transected, total at treated levels } \\
\hline Right & $43 \%$ & $47 \%$ & $42 \%$ \\
\hline Left & $44 \%$ & $50 \%$ & $38 \%$ \\
\hline \multicolumn{4}{|c|}{ Percentage of dorsal rootlets transected, by level } \\
\hline \multicolumn{4}{|c|}{ Right } \\
\hline S-1 & $40 \%$ & $50 \%$ & $50 \%$ \\
\hline L-5 & $50 \%$ & $66 \%$ & $40 \%$ \\
\hline $\mathrm{L}-4$ & $45 \%$ & $30 \%$ & $25 \%$ \\
\hline L-3 & $30 \%$ & $40 \%$ & $45 \%$ \\
\hline $\mathrm{L}-2$ & $50 \%$ & $50 \%$ & $50 \%$ \\
\hline \multicolumn{4}{|l|}{ Left } \\
\hline S-1 & $50 \%$ & $66 \%$ & $50 \%$ \\
\hline$L-5$ & $66 \%$ & $66 \%$ & $55 \%$ \\
\hline$L-4$ & $40 \%$ & $33 \%$ & $35 \%$ \\
\hline$L-3$ & $40 \%$ & $33 \%$ & $0 \%$ \\
\hline L-2 & $25 \%$ & $50 \%$ & $50 \%$ \\
\hline
\end{tabular}

$\mathrm{PVL}=$ periventricular leukomalacia; VPS = ventriculoperitoneal shunt.

sit on the ground for a few minutes until the sensation went away and she could get up again. This sensation in the legs usually occurred after strenuous exercise or at the end of the day, although it could also appear spontaneously. The sensation was progressive, and 1 year after SDR it occurred daily, alternating between the left and right legs. One time she fell downstairs after the sensation occurred while climbing the stairs. The sensory symptom was further investigated by means of MRI of the brain and the spinal cord, which showed no abnormalities. Amitriptyline (10 mg) was prescribed ante noctem, but the drug was quickly discontinued due to the side effect of nausea. Instead, gabapentin was prescribed $(100 \mathrm{mg}, 3$ times a day; total daily dosage of $300 \mathrm{mg}$ ) in combination with psychotherapy to help her cope with the limitations in daily life. During this period, the sensory symptoms were reduced but the sudden falls still occurred several times a day. Within 2 months of initiating gabapentin therapy, the sudden falls decreased in frequency and finally disappeared. Gabapentin treatment was discontinued after 3 months of treatment, and the abnormal sensation in the legs returned, but only infrequently. When this sensation did occur, the patient stopped walking for a moment, which made the sensation disappear, and she no longer had sudden falls. Eighteen months after discontinuation of gabapentin, in 2015 , the sensory symptoms and sudden falls had returned, and gabapentin was prescribed again.

\section{Discussion}

We report sudden falls as a persistent complication of SDR in childhood in 3 cases. Triggers for the sudden falls were performing dual tasks, transition from sitting to standing, running, sensory sensations in the legs, strenuous exercise, or a fright. The exact mechanism of action by which the sudden falls originate is unknown.

Hyperesthesia often occurs shortly after SDR, as a radiculopathy. To alleviate this hyperesthesia, we use a protocol that includes perioperative treatment with orally administered amitriptyline $(1 \mathrm{mg} / \mathrm{kg}$, to a maximum of $10 \mathrm{mg}$ a day) and intravenously administered morphine, s-ketamine, and paracetamol. In most patients, the hyperesthesia disappears and the amitriptyline is stopped after 1 week. In our 3 cases, hyperesthesia, mostly of the sole of the foot, persists and they suffer from sudden falls, spontaneously or after strenuous exercise.

We therefore hypothesized that sudden falls are caused by an inhibition reflex of the muscles in the legs, as an abnormal reaction to a sensory stimulus that is perceived as an enhanced sensory stimulus in patients with hyperesthesia. To suppress the hyperesthesia, we started treatment with gabapentin in 2 of the 3 cases, and the patients subsequently experienced a reduction in the frequency of falls. Based upon this observation, we think that gabapentin might reduce the muscle inhibition reflex and thereby 
prevent the sudden falls. Gabapentin is an analog of the neurotransmitter gamma-aminobutyric acid (GABA), but it does not bind to GABA receptors and is not converted into a GABA agonist. Although the exact mechanism of action of gabapentin is not clear, it is known to reduce neuronal sensitivity by binding to an auxiliary subunit $(\alpha 2-\delta)$ of voltage-gated calcium channels on central neurons. In addition, it reduces the release of various neurotransmitters, such as glutamate, noradrenaline, and substance $\mathrm{P}$.

Because not all children with hyperesthesia experience (persistent) sudden falls, and because hyperesthesia may play less of a role in the other triggers (performing dual tasks, transition from sitting to standing, running, strenuous exercise, or a fright), the sudden falls may have a multifactorial cause. In view of the small number of cases and the absence of data for a control group, we cannot draw quantitative conclusions. Nevertheless, since all of the patients who underwent SDR at VU University Medical Center had an annual follow-up evaluation performed by the same single physician, we can make some general descriptive observations. First, the persistent sudden falls were experienced by patients with MRI abnormalities as well as by those with normal MRI results, so there is probably no relation between MRI findings and the risk of having persistent sudden falls after SDR.

Second, one might hypothesize that patients who are overweight have a higher risk of falling due to the combination of being overweight and having limited lowerextremity muscle strength, which is common in patients with CP. ${ }^{12}$ However, all of our patients who experienced sudden falls had a body weight within normal range. No abnormal changes in BMI occurred before development of the sudden falls, so BMI is not likely to be a factor.

Third, all patients had fairly good muscle strength, since they met the inclusion criteria for SDR: at least being able to crawl, sit independently for more than $10 \mathrm{sec}-$ onds, maintain a raised knee position (support for balance allowed), and squat at least 7 times. However, while calf muscle strength is especially important in preventing falls, the strength of soleus and gastrocnemius muscles is not specifically examined before SDR. So weakness of the calf muscles may still play a role.

Another factor that also may contribute to the sudden falls is suboptimal proprioception due to the reduced sensory input as a result of SDR.

Lastly, younger age and developmental ability in the preoperative phase compared with developmental ability in the postoperative phase may be a confounder. However, even in children with a similar young age at SDR there is a difference in occurrence of the sudden falls; for most of these children the sudden falls fully resolve within months after SDR, but some of the children continue to experience these sudden falls after SDR or develop them later on. Still, the effect of age and developmental ability before and after SDR cannot fully be eliminated. In the future, larger longitudinal studies should allow the analysis of groups of children with a similar (young) age at SDR.

We hope that this report and discussion of sudden falls as a persistent complication of SDR will encourage others to record this complication so as to improve our understanding of the phenomenon.

\section{References}

1. Bolster EAM, van Schie PE, Becher JG, van Ouwerkerk WJ, Strijers RL, Vermeulen RJ: Long-term effect of selective dorsal rhizotomy on gross motor function in ambulant children with spastic bilateral cerebral palsy, compared with reference centiles. Dev Med Child Neurol 55:610-616, 2013

2. Fink JK: Hereditary spastic paraplegia: clinico-pathologic features and emerging molecular mechanisms. Acta Neuropathol 126:307-328, 2013

3. Grunt S, Becher JG, van Schie P, van Ouwerkerk WJ, Ahmadi M, Vermeulen RJ: Preoperative MRI findings and functional outcome after selective dorsal rhizotomy in children with bilateral spasticity. Childs Nerv Syst 26:191-198, 2010

4. Himmelmann K, Uvebrant P: The panorama of cerebral palsy in Sweden. XI. Changing patterns in the birth-year period 2003-2006. Acta Paediatr 103:618-624, 2014

5. Langerak NG, Lamberts RP, Fieggen AG, Peter JC, Peacock WJ, Vaughan CL: Selective dorsal rhizotomy: long-term experience from Cape Town. Childs Nerv Syst 23:1003-1006, 2007

6. Park TS, Johnston JM: Surgical techniques of selective dorsal rhizotomy for spastic cerebral palsy. Technical note. Neurosurg Focus 21(2):e7, 2006

7. Peacock WJ, Staudt LA: Spasticity in cerebral palsy and the selective posterior rhizotomy procedure. J Child Neurol 5:179-185, 1990

8. Rosenbaum P, Paneth N, Leviton A, Goldstein M, Bax M, Damiano D, et al: Definition and classification of cerebral palsy April 2006. Dev Med Child Neurol Suppl 109:8-14, 2007 (Erratum in Dev Med Child Neurol 49:480, 2007)

9. Sanger TD, Delgado MR, Gaebler-Spira D, Hallett M, Mink JW: Classification and definition of disorders causing hypertonia in childhood. Pediatrics 111:e89-e97, 2003

10. van Schie PE, Schothorst M, Dallmeijer AJ, Vermeulen RJ, van Ouwerkerk WJ, Strijers RL, et al: Short- and long-term effects of selective dorsal rhizotomy on gross motor function in ambulatory children with spastic diplegia. J Neurosurg Pediatr 7:557-562, 2011

11. van Schie PE, Vermeulen RJ, van Ouwerkerk WJ, Kwakkel G, Becher JG: Selective dorsal rhizotomy in cerebral palsy to improve functional abilities: evaluation of criteria for selection. Childs Nerv Syst 21:451-457, 2005

12. Wiley ME, Damiano DL: Lower-extremity strength profiles in spastic cerebral palsy. Dev Med Child Neurol 40:100107,1998

\section{Disclosures}

Dr. Vermeulen reports receipt of lecture expenses from Medtronic.

\section{Author Contributions}

Conception and design: Grootveld, van Schie, Becher. Drafting the article: Grootveld. Critically revising the article: van Schie, Buizer, Vermeulen, van Ouwerkerk, Strijers, Becher.

\section{Correspondence}

Laura R. Grootveld, Department of Rehabilitation Medicine, VU University Medical Center, PO Box 7057, Amsterdam 1007 MB, The Netherlands.email: 1.grootveld@vumc.nl. 